\title{
Riflessioni sulla diffusione delle costruzioni scisse nell'italiano giornalistico odierno a partire dalla loro manifestazione nei lanci di agenzia in italiano e in inglese*
}

\author{
Anna-Maria DE CESARE \\ Università di Basilea $^{1}$ \\ anna-maria.decesare@unibas.ch
}

Recibido: 20/07/2012

Aceptado: 30/09/2012

\section{RIASSUNTO}

L'obiettivo di questo lavoro consiste nel riflettere sulla diffusione delle costruzioni scisse nell'italiano contemporaneo, in particolare giornalistico, seguendo l'ipotesi di una possibile contaminazione tra italiano e inglese. Per questo studio abbiamo scelto di soffermarci sulla scrittura dei lanci di agenzia online, prodotti dalle agenzie di stampa per i mass media e ora diffusi in massa in rete. La scelta di soffermarsi sui testi dei lanci di agenzia si giustifica con il fatto che, rispetto agli altri testi giornalistici, questi testi hanno uno statuto primario: non solo per quanto riguarda il contenuto trasmesso, ma anche a livello di lingua, perché i lanci formano in particolare "l'intelaiatura morfosintattica" di altri testi giornalistici e possono dunque innescare delle (nuove) abitudini linguistiche o contribuire a diffondere dei tratti linguistici in diffusione. Alla luce dei dati quantitativi e qualitativi proposti in questo studio sia per i dati dell'italiano sia in chiave contrastiva con i dati dell'inglese, l'ipotesi della diffusione delle costruzioni scisse nell'italiano giornalistico odierno a partire da una contaminazione dell'inglese a livello dei testi prodotti dalle agenzie di stampa non sembra però attendibile.

Parole chiavi: costruzioni scisse, analisi contrastiva italiano-inglese, lanci di agenzia, italiano giornalistico, contatto linguistico.

* Questo lavoro si iscrive in un progetto di ricerca finanziato dal Fondo Nazionale Svizzero per la Ricerca Scientifica che si intitola Italian Constituent Order in a Contrastive Perspective (ICOCP) (PP00P1 133716/1).

${ }^{1}$ Anna-Maria De Cesare, Linguistica italiana, Maiengasse 51, 4056 Basel. 


\title{
Some thoughts on the spread of cleft constructions in contemporary journalistic Italian on the basis of their occurrence in news releases written in Italian and in English
}

\begin{abstract}
The goal of the paper is to discuss the hypothesis of the spreading of cleft constructions in contemporary Italian through a possible contamination with the English language. The discussion is centered on news releases, a text type produced by news agencies for the media (both oral and written). If we have chosen to focus on texts produced by news agencies, it is because news releases can be considered to have a primal status: not only in terms of the content conveyed, but also as far as the language goes. From a linguistic point of view, news releases form in particular "the morphosyntactic canvas" of other journalistic texts and can thus trigger (new) linguistic habits or contribute to further spreading linguistic features that are already on the spread. In light of the quantitative and qualitative data proposed in this study for Italian and in a contrastive perspective with English, the hypothesis of the spread of Italian cleft constructions in contemporary journalistic texts via a contamination with English in the texts produced by news agencies does not seem plausible.
\end{abstract}

Keywords: cleft constructions, contrastive analysis Italian-English, news releases, journalistic Italian, language contact

SOMMARIO: 1. Introduzione. 2. Breve caratterizzazione dei lanci di agenzia. 2.1. Caratteristiche tipologiche. 2.2. La diffusione dei lanci nel giornalismo odierno. 3. Breve caratterizzazione delle costruzioni scisse. 4. Corpus di lavoro. 5. Le costruzioni scisse nei lanci in italiano. 5.1. Dati quantitativi e forma delle costruzioni scisse. 5.2. Funzioni delle costruzioni scisse. 6. Le costruzioni scisse nei lanci in italiano e in inglese. 6.1. Dati quantitativi e forma delle costruzioni scisse. 6.2. Funzioni delle costruzioni scisse. 7. Osservazioni conclusive

\section{INTRODUZIONE}

A partire da uno spoglio di quotidiani italiani pubblicati lungo l'arco di un secolo, Bonomi 2002 (N. 83, p. 44) osserva che le costruzioni scisse del tipo illustrato di seguito (gli esempi sono dell'Autrice) sono sporadiche all'inizio del Novecento, sono meno infrequenti negli anni 1930 e diventano molto comuni nel dopoguerra:

(1) È stato appunto durante questo controllo che è venuto alla luce (Il Giornale, 3.5.1965)

(2) questa questione è soprattutto dai meridionali che deve essere risolta (Il Mattino, 20.1.1930) 
(3) La verità è che il solo che avrebbe interesse [...] è il governo (Il Resto del Carlino, 17.11.1953)

L'obiettivo principale del presente contributo consiste nel capire meglio le modalità della diffusione delle costruzioni scisse nell'italiano contemporaneo, in particolare nei mass media odierni. Due sono le spiegazioni generalmente proposte nella bibliografia.

La prima spiegazione riconduce la diffusione delle scisse ad una tendenza generale ad assorbire strutture tipiche del parlato nella lingua scritta; le scisse, assieme ad altre costruzioni, contribuiscono così ad abbassare il tono dei testi scritti nei quali compaiono e a formare una nuova varietà di italiano contemporaneo, che si chiama, proprio in riferimento diretto al suo grado di formalità, italiano dell'uso medio. Questa proposta, articolata come è ben noto in particolare in Sabatini (1985), si ritrova in numerosissimi lavori successivi. Basta qui citarne due dei più recenti:

Un altro segnale dell'apertura [della lingua dei giornali] verso il parlato sono i costrutti di sintassi marcata, nei quali, cioè, l'ordine lineare delle parole viene modificato per mettere in rilievo un elemento del discorso cui si vuol dare particolare risalto. [...] frasi scisse. (Gualdo 2007: 111)

«Dove va la lingua italiana?» Se lo chiedono in tanti. Va dove la porta chi la parla e chi la scrive. Una direzione molto evidente (basta leggere con attenzione il giornale) è per esempio l'assestamento generale dell'italiano verso una norma orientata sempre di più sull'oralità. Tra i tanti esempi da addurre, si osservi soltanto come gli abbia pian piano sostituito quasi del tutto il loro ed egli sia usato sempre meno di fronte a lui soggetto; [...] di tono colloquiale le frasi scisse, le molte dislocazioni a sinistra, ora dell'oggetto [...], o del partitivo [...], ora del locativo [...]. (Beccaria 2010: 101, il corsivo sottolineato è nostro)

Lo osservava già Maurizio Dardano in un suo saggio che tratteggia il Profilo dell'italiano contemporaneo:

Da un punto di vista sociolinguistico possiamo dire che la scrittura giornalistica riprende costruzioni con ordine marcato degli elementi, presenti di solito nel parlato, al fine di assecondare la tendenza verso uno standard più vicino a livelli medi. [...] Frase scissa: c'è un nuovo protagonista sulla scena politica; C'è un problema che dobbiamo subito affrontare; È stato lo stesso presidente ad aprire i lavori; Ad aprire i lavori è stato lo stesso presidente. [...] La frase scissa è frequente nel parlato-scritto del telegiornale (Dardano 1994: 401, il corsivo sottolineato è sempre nostro)

In realtà il discorso è molto più complesso: non solo perché - come ha notato del resto lo stesso Sabatini (1990) e ha poi mostrato a partire da una ricca base empirica il lavoro di Roggia (2012) - le scisse erano presenti già nei testi di italiano antico, 
ma anche perché non tutte le scisse sono da ricondurre in blocco al parlato (dialettale, regionale o panitaliano che sia). Il tono colloquiale associato alle scisse dipende infatti strettamente dalla forma della costruzione, cioè dal sottotipo di scissa considerato. Basta qui menzionare un aspetto ormai noto e cioè che le scisse implicite (è Stella ad aver telefonato / ad aver telefonato è Stella) sono più tipiche dello scritto che del parlato, mentre che le scisse interrogative (chi è che ha telefonato? dov'è che è andato? ecc.), le scisse inferenziali (è che / non è che), le scisse temporali cosiddette spurie (del tipo sono anni che non lo vedo, e descritte in particolare da Benincà 1978) e alcune forme di frasi pseudoscisse sono decisamente più tipiche della lingua parlata (questi dati emergono in molti studi, tra $\mathrm{i}$ quali Berretta 1994b e Roggia 2008, 2009: 166).

La seconda spiegazione che si dà alla diffusione delle scisse - generalmente dal Settecento in poi, e pensando in modo specifico alle scisse vere e proprie (del tipo è Stella che è partita in vacanza) - fa invece leva sulla contaminazione dell'italiano con un'altra lingua: si è parlato dapprima del francese (cfr. Fornaciari (1881), Migliorini (21963: 543) e il commento di Serianni (1988) [ripreso in tutte le ristampe successive], che cerca di sfatare un'idea ancora molto diffusa almeno al momento della stesura della grammatica ${ }^{2}$ ), ora si parla piuttosto dell'inglese (cfr. per esempio D'Achille et al. (2005: 30) $)^{3}$.

L'obiettivo di questo lavoro consiste nel riflettere sulla seconda spiegazione data alla diffusione delle costruzioni scisse in italiano contemporaneo, vale a dire sulla possibile contaminazione tra italiano e inglese. Per questo studio abbiamo scelto di soffermarci su un tipo di testo particolarmente appropriato - almeno così ci sembra - per affrontare la questione. Si tratta della scrittura dei lanci di agenzia (o, con un

${ }^{2}$ Cfr. Serianni (1998: XIV.81): «La frase scissa ricevette un forte impulso dall'influsso del francese nel Settecento (cfr. Migliorini 1963a: 543), benché anche l'italiano antico conoscesse costrutti molto simili (cfr. Durante 1981: 204-205). Oggi è assai frequente nel parlato e nello scritto di qualsiasi livello: ormai del tutto spente le riserve dei puristi che parlavano di "noiosa tiritera" per il costrutto è a voi che parlo, paragonandolo a "uno starnuto che dia il tono al periodo" (cosí Romanelli 1910: 130)».

${ }^{3}$ Sull'influsso che esercita la lingua inglese sulle strutture dell'italiano odierno esiste ormai una bibliografia molto nutrita, dedicata soprattutto alla tipologia dei prestiti e dei calchi lessicali (cfr., per limitarci ai volumi, Klajn 1972, Sullam Calimani 2003 e Bombi 2005). Molto meno è stato detto sull'influsso dell'inglese sulle strutture sintattiche dell'italiano (su questo aspetto si vedano per esempio la tesi di dottorato di Grasso (2007), le considerazioni proposte in De Cesare (2009) e (2010) su diversi tipi di ordini dei costituenti e i lavori che Degano (2005), Cortelazzo (2007) e da ultimo Brianti (2010) dedicano alla perifrasi progressiva). A livello sintattico, la questione dell'influsso e del contatto linguistico è molto complessa e controversa. Delicata è in particolare la questione delle modalità del contatto: il contatto avviene attraverso testi tradotti (come suggeriscono per esempio Alfieri et al. (2003) a partire da dati relativi al doppiaggio televisivo e Degano (2005) a partire da dati dello scritto) oppure può avvenire anche attraverso testi originali? Avviene attraverso testi massmediatici e/o specializzati? Pensiamo qui naturalmente all'ambito scientifico, per cui si apre anche la questione della diglossia (aspetto sul quale sono cruciali i lavori di Calaresu 2006 e 2011). 
termine più tradizionale, dispacci di agenzia), prodotti dalle agenzie di stampa per $\mathrm{i}$ mass media (sulle modalità di confezione degli articoli e sui lanci stessi, cfr. per esempio Lepri 1982 e Papuzzi 2003), e ora diffusi in massa online (all'interno di una rubrica che prende un nome spesso inglese: notizie flash sul sito dell'Unità, ultime news sul sito dell' ANSA, ATS News sul sito del Giornale del Popolo; alcuni esempi si trovano in Appendice a questo lavoro). La scelta di soffermarsi sui testi dei lanci di agenzia si giustifica con il fatto che, rispetto agli altri testi giornalistici, questi testi hanno uno statuto per così dire "primario": non solo per quanto riguarda il contenuto trasmesso (le note d'agenzia, come si sa, sono la fonte di molti altri testi orali e scritti diffusi dai media), ma anche a livello di lingua, perché i lanci formano in particolare - secondo i termini di Palermo (1997: 202) - "l'intelaiatura morfosintattica" di altri testi giornalistici e possono dunque innescare delle (nuove) abitudini linguistiche (così ancora secondo Palermo 1997: 187). Questo dato di fatto è stato riconosciuto e preso molto sul serio dai giornalisti di agenzia stessi, in particolare da quelli dell'ANSA. Nel loro Vademecum di giornalismo (ANSA 1992) si legge infatti: «le soluzioni adottate dall' ANSA possono trasferirsi - direttamente o attraverso il giornale - nella società nazionale. È una responsabilità di cui è bene tener conto».

Uno studio che verte sulla lingua dei lanci di agenzia - ancora poco nota ${ }^{4}-\mathrm{si}$ giustifica anche a partire dalla constatazione che questi testi sono molto presenti nel panorama dei media elettronici odierni, nei quali sono anzi in diffusione, su scala molto ampia e presso un pubblico molto vario. Infatti, se una ventina d'anni fa i lanci di agenzia erano poco visibili, poco diffusi e poco noti in particolare al grande pubblico (perché occupavano spazi marginali della pagina dei giornali cartacei), stanno ormai acquistando un posto centrale sia nell'ambito dei giornali online, sia in quello dei giornali cartacei. I motivi di questo successo sono da ricondurre almeno all'avvento di Internet, alla fortuna che stanno avendo negli ultimi anni i giornali gratuiti e a quella che conoscono gli ultimi mezzi di comunicazione di massa alla moda, gli smartphone, il cui schermo si presta soprattutto alla fruizione di testi brevi quali sono appunto i lanci.

Prendendo le mosse da queste riflessioni generali, le domande alle quali si cercherà di dare una risposta nelle pagine che seguono sono in particolare quattro: (i) le costruzioni scisse compaiono già nei lanci di agenzia, cioè nei testi che fondano la lingua dei mass media?; (ii) con che frequenza, in che forma, in che momenti della notizia (nel discorso riportato o nel testo scritto dal giornalista di

${ }^{4}$ La bibliografia sull'argomento, almeno in ambito italiano, non è molto ampia. Sulle caratteristiche linguistiche (ortografiche, lessicali, interpuntive, sintattiche, morfologiche, stilistiche) di questo tipo di testo si possono citare soprattutto gli studi di Palermo (1995) e (1997) (il secondo lavoro menzionato contiene alcune osservazioni di rilievo sulle dislocazioni); per una descrizione fine della sintassi dei lanci (a livello di clausola e di periodo), si rinvia invece al lavoro di De Cesare / Baranzini (in stampa). 
agenzia, all'inizio o alla fine della notizia ecc.) e con quali funzioni?; (iii) quali sono i punti comuni e le divergenze nell'impiego delle costruzioni scisse nei lanci in italiano e in inglese?; infine, e soprattutto, (iv) a partire dalle risposte fornite al punto (iii) è possibile riconoscere un influsso dell'inglese sull'italiano, ipotizzando per esempio la presenza di testi o porzioni di testi tradotti dall'inglese all'italiano in modo invisibile, cioè non dichiarato? Quest'ultima domanda è per noi cruciale in quanto potrebbe essere un importante tassello per spiegare la diffusione delle scisse nell'italiano massmediatico odierno (cioè all'interno dei giornali cartacei, elettronici, e del linguaggio radiotelevisivo).

A queste domande si risponderà in modo puntuale nella parte conclusiva di questo lavoro alla luce dei risultati ottenuti sulla base di un'analisi corpus-based di 623 lanci in italiano e in inglese pubblicati online (nell'ultimo trimestre del 2011). Il lavoro è organizzato più precisamente nel modo seguente: si proporrà dapprima una breve caratterizzazione dei lanci di agenzia $(\S 2)$, delle costruzioni sintattiche di cui ci occupiamo qui $(\S 3)$, così come una breve descrizione del corpus analizzato per rispondere alle domande poste in (i)-(iv) (§ 4); si passa poi alla descrizione dei risultati ottenuti, concentrandoci in un primo momento sull'impiego delle costruzioni scisse nei lanci in italiano $(\S 5)$, poi sul loro impiego nei lanci in italiano a confronto con quelli in inglese $(\S 6)$.

\section{BREVE CARATTERIZZAZIONE DEI LANCI DI AGENZIA}

\subsection{Caratteristiche tipologiche}

Prima di descrivere le specificità tipologiche dei lanci di agenzia, una breve nota terminologica: oltre al termine lancio di agenzia, si impiega anche quello di dispaccio di agenzia (termine che risale all'Ottocento), di nota d'agenzia (Palermo 1997: 185), di notizia d'agenzia o, con un termine inglese più generico, di news. Il termine lancio o dispaccio (secondo le indicazioni di Palermo 1995: 93) vale solo per le notizie che l'agenzia invia ai suoi clienti (media di varia natura e clienti del settore privato). Le notizie che il giornalista d'agenzia invia all'agenzia stessa sono invece chiamate originale. Da notare ancora, ma si tratta qui di una cosa nota, che i lanci di agenzia non coincidono con i comunicati stampa. I lanci sono redatti da giornalisti professionali che lavorano per un'agenzia di stampa, mentre che $i$ comunicati stampa sono scritti da professionisti della comunicazione (sono i cosiddetti addetti stampa, fr. attachés de presse) che lavorano in uffici stampa specializzati di istituzioni pubbliche (governi, università ecc.) o private (ditte ecc.).

I lanci di agenzia sono prodotti dalle cosiddette agenzie di stampa, oggi chiamate sempre più spesso agenzie d'informazione, perché non si rivolgono più soltanto alla stampa (ma anche, come si diceva, a clienti del settore privato). In Italia, in modo del tutto anomalo rispetto ad altri paesi occidentali, si contano varie 
agenzie di stampa; tra queste si possono menzionare le agenzie seguenti: ANSA, creata nel 1945 e oggi una delle più grandi agenzie a livello mondiale (produce, secondo i dati riportati da Gualdo (2007: 93), 350.000 parole al giorno), TMNews, Adnkronos, AGI, Italpress e ASCA. In Svizzera è attiva l'agenzia ATS, in Francia l'Agence France Presse (AFP), negli Stati Uniti l'Associated Press (AP) ecc.

Da un punto di vista tipologico, o varietistico, i lanci di agenzia rientrano nella stessa macrocategoria dei testi giornalistici, fanno cioè parte - secondo la proposta di Sabatini (1999) - dei testi informativi. La definizione generale che Sabatini (1999) propone per i testi informativi non si applica tuttavia in modo perfetto ai testi dei lanci di agenzia. I testi informativi hanno infatti «[f]unzione informativa, basata sull'intenzione di mettere genericamente a disposizione ("divulgare") informazioni, perlopiù sommarie e approssimative» (Sabatini 2011 [1999]: 195). Ora, è chiaro che l'obiettivo comunicativo dei lanci è ben altro: qui si tratta di diffondere notizie il più fedelmente e precisamente possibile. Cruciale è anzi, come avremo modo di costatare sulla base del corpus analizzato, la fonte delle informazioni riportate. Da notare poi che, contrariamente ad altri tipi di testo informativo (opere divulgative, come i manuali), nei lanci di agenzia sono di solito assenti i momenti esplicativi. Quello che conta nei lanci è riportare le informazioni; la loro spiegazione è invece il compito di un altro mediatore della notizia: il giornalista della carta stampata, del telegiornale o che scrive per la rete.

I lanci di agenzia non hanno tutti la stessa forma, in particolare la stessa lunghezza. A un primo livello, si possono infatti riconoscere due tipi di lanci: (i) il flash (o flash di agenzia o notizia flash), che coincide con la prima notizia, generalmente molto breve, diffusa da un'agenzia di stampa, come per esempio:

(4) ANSA 6-09-01, 12:12:23 - ATLETICA: MARCIA: 20KM: RECORD MONDIALE PER IVANOVA

- BRISBANE, 6 SET - Nuovo primato del mondo nella marcia ai Goodwill Games australiani: la russa Ivanova ha vinto la gara in 1 ora, 26'52'3 cancellando il precedente record che apparteneva alla portoghese Feito. (es. tratto da Gualdo 2007: 95)

e (ii) il take, che segue generalmente il flash, è più lungo del primo (non supera però, secondo Papuzzi (2003: 37), le 24 righe di testo) ed è tipicamente costruito secondo le regole delle cinque $w$ (chi / che cosa, dove, quando, come, perché):

(5) $\quad \underline{\text { ANSA.it }}>\underline{\text { Top News }}>$ News

Usa: decine animali feroci fuggiti da zoo

Morto proprietario fattoria, si disperdono tigri, leoni, orsi

19 ottobre, 15:26

(ANSA) - NEW YORK, 19 OTT - Allarme rosso in una contea dell'Ohio dove decine di animali feroci, tra cui tigri, leoni ghepardi, orsi e lupi sono scappati 
da una fattoria privata il cui proprietario e' stato trovato morto. Lo sceriffo ha deciso la chiusura delle scuole e ha chiesto alla popolazione di rimanere barricata in casa, mentre lui e i suoi vice sono impegnati in un vero e proprio safari. Sembra che in tutto siano fuggiti 48 animali. Oltre alle belve ci sono cammelli, giraffe e altri animali esotici. (2011)

In questo lavoro ci occupiamo solo dei take (di cui si propongono altri esempi alla fine del lavoro), e di questi ci concentriamo unicamente sulle parti di testo, ovvero sul titolo e sul testo vero e proprio.

\subsection{La diffusione dei lanci nel giornalismo odierno}

Come si diceva nell'introduzione, se una ventina d'anni fa i lanci di agenzia erano poco visibili, poco diffusi e poco noti in particolare al grande pubblico, con l'avvento di Internet, la fortuna dei giornali gratuiti e degli ultimi supporti di comunicazione di massa, i lanci stanno oggi acquistando un posto centrale nell'ambito della scrittura giornalistica. Li si trova ormai non solo su gran parte delle pagine principali dei quotidiani online (nazionali, regionali e locali, nella versione gratuita dei quotidiani a pagamento o in quella elettronica dei giornali gratuiti), sotto il titolo della testata o in un riquadro che occupa caratteristicamente uno spazio in alto a destra della pagina, ma anche in siti che non assomigliano né da vicino né da lontano a giornali "tradizionali" e nei siti delle agenzie di stampa stesse. Il rilievo dei lanci è incrementato dal fatto che sono molto spesso proposti come testi a scorrimento ${ }^{5}$.

La fortuna dei testi di agenzia va sicuramente ricondotta a quella che stanno avendo, in questi ultimi anni, altri testi brevi, come gli sms, i messaggi elettronici, i messaggi delle chat ecc. ${ }^{6}$ : nella nostra società dell'informazione, i lanci sono un tipo di testo di facile codifica e di facile consumo (un tipo di testo "usa e getta", secondo l'efficace formula di Antonelli 2007).

${ }^{5}$ La presentazione dei lanci sui siti dei quotidiani online è un aspetto che cambia però in modo rapido. Il quadro tracciato in De Cesare \& Baranzini (in stampa) a partire dalla consultazione di vari siti di quotidiani online avvenuta il 20.10.2011 non è in effetti più del tutto valido: una visita in data del 16.6.2012 ha per esempio permesso di osservare che sul sito di lastampa.it è scomparso il newsticker sotto il nome del giornale; ora la rubrica Ultime News compare a mezza pagina e propone, all'interno di un riquadro, tre notizie a carattere fisso (non più a scorrimento).

${ }^{6}$ A questi testi brevi a noi ormai relativamente ben noti, si affiancano altre tipologie di testi brevi, forse meno noti: si pensa in particolare ai testi che compongono la Diretta sul sito della Repubblica, che superano raramente dieci righe. 


\section{BREVE CARATTERIZZAZIONE DELLE COSTRUZIONI SCISSE}

Secondo la definizione ormai classica di Lambrecht (2001), ripresa per l'italiano da Roggia (2009), le costruzioni scisse sono strutture sintattiche copulative biclausali, che hanno una semantica specificativa (o identificativa) e che servono alla messa in rilievo di un'informazione, la quale coincide generalmente con l'elemento scisso.

La famiglia delle costruzioni scisse include almeno le quattro strutture sintattiche seguenti (sulla base di Roggia $2009^{7}$ ): le frasi scisse, come in (6), le frasi scisse inverse, del tipo (7), le pseudoscisse (8) e le pseudoscisse inverse (9):

(6) è Maria che vuole studiare le scisse

(7) a voler studiare le scisse è Maria

(8) chi/quella che/la persona che vuole studiare le scisse è Maria

(9) MARIA è la persona che vuole studiare le scisse

In questo studio abbiamo tenuto conto solo delle scisse del tipo (6)-(8), abbreviate rispettivamente con S, Sinv. e PS. Per via della distribuzione dell'elemento scisso in posizione finale della costruzione, raggrupperemo a volte le Sinv. e le PS in una classe che chiameremo scisse a focus finale (sulla classificazione delle scisse, cfr. De Cesare in prep.), senza cioè estendere l'etichetta di frase pseudoscissa ad includere anche la scissa inversa (come nei lavori di Berretta 1994a, di Gil 2004 e di De Cesare 2005).

A questa caratterizzazione sintattico-semantica delle costruzioni scisse si può aggiungere un dato di carattere tipologico, e cioè che non tutte le lingue sono dotate di scisse e/o usano queste costruzioni in misura eguale (questa idea è diffusa soprattutto nei lavori di stampo tipologico, cfr. Jespersen 1937, Miller 2006, Filppula 2009). Si ritiene infatti che le lingue che hanno un ordine libero o relativamente libero dei costituenti, come il tedesco e le lingue slave, non hanno bisogno di queste costruzioni per ottenere effetti semantico-pragmatici simili alle scisse e dunque non usano o non usano spesso queste costruzioni; in modo speculare, si ritiene poi che le lingue che hanno un ordine (relativamente) rigido dei costituenti, come il francese e l'inglese, devono invece ricorrere a queste costruzioni per mettere a fuoco un'informazione. Questo spiega perché nelle lingue come il francese e l'inglese le costruzioni scisse sono più standard e più diffuse che in tedesco e nelle lingue slave ${ }^{8}$.

\footnotetext{
${ }^{7}$ Su queste costruzioni, cfr. tra molti altri lavori quelli di Berretta (1994a, 2002), Panunzi (2009), Roggia (2009) e De Cesare (2011).

${ }^{8}$ Come si sarà notato, non abbiamo detto nulla sulla prosodia. Questo perché il nostro interesse ruota attorno ai testi scritti, dove non ci sono indicazioni (affidabili, sistematiche e complete) di tipo intonativo.
} 
Il caso dell'italiano è diverso da quello delle altre lingue menzionate. Si tratta infatti di una lingua alla quale si riconosce una grande libertà nell'ordinamento dei costituenti (anche se c'è chi, come Sornicola (1994), considera l'italiano una lingua a ordinamento semi-libero dei costituenti). Al tempo stesso, i lavori che hanno descritto le proprietà centrali dell'italiano cosiddetto dell'uso medio o neo-standard (penso qui in particolare ai lavori di Francesco Sabatini e di Gaetano Berruto citati in bibliografia, ma cfr. anche D'Achille 2003) osservano che le scisse sono un tratto sintattico diagnostico di questa varietà d'italiano contemporaneo.

Alla luce di queste osservazioni ci si aspetterebbe dunque di trovare delle differenze almeno per quanto riguarda la frequenza d'impiego delle scisse (delle scisse vere e proprie, ma anche degli altri tipi di scisse) nel corpus di lanci in italiano e in inglese.

\section{CORPUS DI LAVORO}

L'analisi proposta in questo studio si basa sullo spoglio di una sezione della raccolta di testi ICOCP (Italian Constituent Order in a Contrastive Perspective), un corpus di scrittura giornalistica elettronica, plurilingue (italiano, francese, inglese, tedesco) e comparabile, creato all'università di Basilea con il sostegno del Fondo Nazionale Svizzero per la Ricerca Scientifica.

Più precisamente, il corpus sul quale si basa il nostro studio è composto da 623 lanci (titolo e testo, per un totale di 113.000 parole) in due lingue (italiano e inglese) raccolti durante l'ultimo trimestre del 2011 direttamente sul sito delle agenzie o su quello di altri organi d'informazione (in genere dalla versione gratuita di quotidiani online $)^{9}$. Il corpus di lanci in italiano è composto da 60.000 parole di sei agenzie (abbiamo raccolto 10.000 parole per ogni agenzia): cinque agenzie italiane (ANSA, Adnkronos, AGI, Italpress, TMNews) e un'agenzia svizzera (ATS). A sua volta, il corpus di lanci in inglese è composto da 53.000 parole di lanci prodotti dall'agenzia americana Associated Press (di seguito $\mathrm{AP}^{10}$, per un totale di 43.000 parole) e dall'agenzia svizzera ATS (10.000 parole). I dati quantitativi relativi al numero di lanci per lingua e alla dimensione (misurata a partire dal numero di parole) delle due sottosezioni del corpus sono più precisamente i seguenti:

\footnotetext{
${ }^{9}$ I lanci raccolti durante quel periodo possono anche avere una data anteriore. Il nostro corpus contiene infatti alcuni (pochi) lanci del 2010.

${ }^{10}$ Ecco una breve autodescrizione dell'agenzia AP, che si cita in versione originale: «Often called the "Marine Corps of Journalism" - always first in and last out - AP reports history in urgent installments, always on deadline. AP staff in 300 locations in more than 100 countries deliver breaking news that is seen or read by half the world's population on any given day. It remains a not-for-profit cooperative, owned by 1.500 U.S. newspapers, which are both its customers and its members». (http://www.ap.org/company/history/ap-history).
} 


\begin{tabular}{|c|c|c|c|}
\hline & It & Ing & Totale \\
\hline N di lanci & 502 & 121 & 623 \\
\hline N di parole & 60.000 & 53.000 & 113.000 \\
\hline
\end{tabular}

Tabella 1. Composizione e dimensione del corpus di lavoro

I dati contenuti nella Tabella 1 permettono di osservare che $i$ lanci in inglese sono generalmente più lunghi di quelli in italiano: i lanci raccolti nel corpus in italiano sono circa tre volte più numerosi di quelli che compongono l'altra sezione del corpus ${ }^{11}$. Un'altra cosa da notare, che però non si vede dai dati della Tabella 1, è che i lanci in italiano hanno una lunghezza variabile: quelli dell'agenzia TMNews e in parte anche quelli dell'agenzia svizzera ATS sono a volte molto più lunghi di quelli delle altre cinque agenzie. Questa osservazione non è banale in quanto potrebbe avere delle ricadute sulla presenza e sulla forma delle costruzioni scisse all'interno dei nostri testi. Più precisamente, nei testi dei lanci più corti potrebbero venire a mancare le scisse a funzione coesiva o di transizione (sulle quali, cfr. in particolare Berretta 2002 e Roggia 2009), che connettono blocchi ampi di testo (quali i capoversi).

\section{LE COSTRUZIONI SCISSE NEI LANCI IN ITALIANO}

\subsection{Dati quantitativi e forma delle costruzioni scisse}

Vediamo dapprima i dati quantitativi relativi al corpus dei soli lanci in italiano (si ricorda che il corpus si compone di lanci tratti da sei agenzie - cinque italiane, una svizzera - e che si è raccolto un numero equivalente a 10.000 parole per ogni agenzia; i dati proposti per ogni agenzia sono dunque quantitativamente comparabili):

\begin{tabular}{|l|c|c|c|c|}
\hline Agenzie / Tipi di scisse & $\mathrm{S}^{12}$ & Sinv. $^{13}$ & PS & TOTALE \\
\hline ANSA & 2 & $3+2$ & 1 & 8 \\
\hline Adnkronos & 2 & $4+2$ & 0 & 8 \\
\hline AGI & 0 & $3+0$ & 2 & 5 \\
\hline
\end{tabular}

11 Per la loro lunghezza, i testi dell'agenzia AP assomigliano per certi versi più ad articoli giornalistici veri e propri che a veri lanci di agenzia.

${ }^{12}$ Abbiamo scartato dal conteggio le scisse temporali del tipo è la prima volta che / it's the first time that (cfr. Roggia 2009: 18), di cui abbiamo due occorrenze nei lanci in italiano e sei in quelli in inglese.

${ }^{13}$ Il primo dato numerico coincide con le scisse inverse verbali, il secondo con le scisse inverse nominali (esempi di questi due tipi di scisse inverse sono date di seguito). 


\begin{tabular}{|l|c|c|c|c|}
\hline Italpress & 2 & $3+1$ & 0 & 6 \\
\hline TMNews & 8 & $4+0$ & 1 & 13 \\
\hline ATS & 0 & $2+0$ & 1 & 3 \\
\hline TOTALE & 14 & $19+5$ & 5 & 43 \\
\hline
\end{tabular}

Tabella 2. Costruzioni scisse nel corpus di lanci in italiano (dati assoluti)

Da questi primi dati quantitativi spiccano gli aspetti seguenti: (i) innanzitutto va osservato che le costruzioni scisse sono presenti nel nostro corpus di lanci (ve ne sono 43 occorrenze in un corpus di 60.000 parole); valutare la loro frequenza d'impiego come alta o bassa è però un'operazione relativamente delicata, soprattutto senza termine di paragone. Ritorneremo dunque su questo aspetto nel paragrafo successivo, dedicato al confronto tra italiano e inglese; (ii) le costruzioni scisse non sono impiegate in modo omogeneo all'interno dei lanci delle sei agenzie prese in considerazione: si ha un massimo di 13 occorrenze nel campione di TMNews e un minimo di 3 occorrenze in quello dell'agenzia svizzera ATS (la media è di 7.2); (iii) a prescindere dalla differenza menzionata al punto (ii), si osserva che il tipo di costruzione scissa che predomina nei lanci dei testi analizzati (con la sola eccezione di TMNews) sono le cosiddette scisse inverse, che si manifestano sia sotto forma verbale (come nell'esempio (10) proposto di seguito), sia sotto forma nominale (cfr. 11), cioè con ellissi della copula):

(10) (ANSA) - ROMA, 7 SET - Avrà "una portata dirompente" sul sistema Giustizia il ddlLussana sul "processo lungo", approvato dal Senato a fine luglio, perché "legittimando" le più varie tattiche dilatorie da parte degli imputati avrà la "capacità di rallentare a dismisura la durata di tutti i procedimenti in corso". $A$ lanciare l'allarme è il plenum del Csm con una risoluzione approvata a larga maggioranza, con il no dei laici del Pdl. $\left(2011^{14}\right)$

(11) Inchiesta trans: il 10/1 prima udienza

A rischiare processo anche 4 carabinieri del ricatto a Marrazzo (ANSA, 7.9.2011, titolo e sottotitolo)

Il secondo tipo di scissa inversa, già rilevato nella bibliografia, è considerato tipico della prosa giornalistica (Berretta 1996, Bonomi 2002: 215). Qui è dunque interessante notare come questa struttura sia già presente nei lanci stessi, cioè nei testi che formano il "canovaccio" degli articoli di giornale.

\footnotetext{
${ }^{14}$ Per mettere in rilievo le costruzioni scisse (e altre strutture sulle quali si concentra la nostra attenzione) abbiamo scelto di usare il corsivo. Questo perché nei lanci di agenzia il grassetto è usato spesso già nel titolo.
} 


\subsection{Funzioni delle costruzioni scisse}

5.2.1. Le costruzioni scisse che compaiono nel nostro corpus di lanci in italiano sono impiegate in modo molto vario. All'interno del ventaglio di possibili funzioni, ve ne è però una particolarmente saliente, perché molto ricorrente, che è associata in modo relativamente fisso all'impiego della frase scissa inversa (verbale o nominale che sia: 9 casi su 24 ovvero nel $38 \%$ dei casi). Questa funzione, illustrata rappresentativamente a partire dagli esempi seguenti (tratti da lanci di diverse agenzie), consiste nell'individuare la fonte dell'affermazione o delle affermazioni riportate di solito immediatamente prima. Nella subordinata che apre la costruzione scissa, il verbo principale all'infinito coincide dunque con un verbo di dire o di opinione e la fonte coincide con il referente codificato dall'elemento scisso che chiude la struttura sintattica:

(12) (AGI) Islamabad - Il capo delle operazioni di Al Qaeda in Pakistan, il saudita Abu Hafs al Shahri, e' stato ucciso nella regione del Waziristan dal missile sparato da un drone americano. A rivelare il nuovo duro colpo subito dall'organizzazione terroristica $\grave{e}$ stato un alto funzionario dell'amministrazione statunitense, che non ha voluto pero' aggiungere dettagli sull'eliminazione del terrorista. (15.9.2011)

(13) Caso Tarantini: imprenditore, Lavitolami impediva rapporto diretto con Berlusconi

Roma, 8 set. - (Adnkronos) - "Il rapporto con il Presidente era un rapporto mio, non era un rapporto indiretto, quindi io volevo riprendere il rapporto con il Presidente e farlo ritornare mio con lui. Lui invece o con una scusa o con un'altra o perché era pericoloso o perché non si poteva, mi negava sempre la possibilità di andare". A dirlo l'imprenditore Gianpaolo Tarantini nell'interrogatorio di garanzia del 3 settembre. (2011)

(14) SIRACUSA (ITALPRESS) - "Ieri mattina nel carcere di Siracusa Domenico Franzese, originario di Afragola (NA), 45enne, detenuto con condanna definitiva e fine pena ottobre 2011 si è impiccato nella sua cella del reparto accettazione dove era stato allocato da alcuni giorni, ovvero dal suo arrivo a Siracusa proveniente dal carcere napoletano di Poggioreale". A dare la notizia è Eugenio Sarno, Segretario Generale della Uil Pa Penitenziari. (16.5.2010)

Da notare poi che in quasi tutti i casi la scissa inversa occupa una posizione fissa all'interno del lancio: la si trova generalmente nel secondo enunciato, subito dopo un enunciato incipitario che riporta sotto forma di discorso diretto (talvolta senza la presenza delle virgolette citanti) l'affermazione centrale del lancio. Accanto a questa modalità di riporto della fonte, che è di gran lunga la più frequente, se ne trovano altre, molto simili, in cui la fonte dell'informazione non è codificata dalla scissa inversa, in particolare dall'elemento scisso, ma compare tra le due parti della 
scissa, per esempio sotto forma di inciso. Si veda il caso seguente, di scissa inversa nominale:

\section{(15) Scommesse: giocate in calo del $25,8 \%$ rispetto all'anno scorso}

Roma, 8 set. (Adnkronos) - Sono stati 49,7 i milioni di euro giocati online ad agosto dagli appassionati delle scommesse sportive, il $25,8 \%$ in meno dello stesso mese 2010 (circa 67 milioni di euro). A influire negativamente sul bilancio del mese appena trascorso - informa l'Agicos- soprattutto il rinvio della prima giornata di campionato a causa dello sciopero dei calciatori. (2011)

All'interno del nostro corpus, vi è un unico caso in cui la funzione di riporto della fonte dell'informazione è svolta da una frase scissa. Ecco l'esempio in questione, in cui si osserva ancora una volta che la fonte (gli stessi inquirenti baresi) è codificata dall'elemento scisso:

(16) Roma, 15 set. (TMNews) -Tanto rumore per nulla: la Procura di Bari ha depositato gli atti dell'inchiesta sulle escort e le temute intercettazioni delle parole di Silvio Berlusconi su ministri e private cittadine italiane e straniere non sono state trascritte. Sono gli stessi inquirenti baresi ad averlo precisato, facendo sapere in una nota che di centomila conversazioni sono state selezionate solo "quelle ritenute rilevanti ai fini dell'accusa". (2011)

Questa modalità sintattico-testuale non è tuttavia l'unica possibile, né quella preferita. Uno studio approfondito della sintassi delle clausole principali dei lanci (cfr. De Cesare \& Baranzini in stampa) ha permesso di rilevare che nei lanci in italiano la fonte dell'informazione è generalmente proposta all'interno di una struttura sintattica marcata più semplice, che presenta l'ordine dei costituenti OVS, in cui $\mathrm{V}$ coincide con un verbo di dire o di opinione al presente o al passato prossimo, $\mathrm{O}$ con un pronome clitico, che precede dunque necessariamente il verbo $\mathrm{e}$ che riprende tutto il contenuto della citazione o delle affermazioni precedenti, e $\mathrm{S}$ con la fonte dell'informazione ${ }^{15}$. Ecco, tra molti altri possibili, alcuni esempi rappresentativi:

(17) Roma, 16 set. (TMNews) - Silvio Berlusconi, allo stato, non si presenterà davanti ai pm di Napoli che lo vogliono sentire come teste nell'inchiesta sul

\footnotetext{
${ }^{15}$ Come abbiamo visto, la scissa inversa si manifesta solo in 24 lanci su 502, e non in tutti i casi ha la funzione di riporto di cui si sta parlando. Della struttura sintattica OVS abbiamo invece contato 151 casi in un corpus di 332 lanci tratti da quattro agenzie di stampa (cfr. De Cesare / Baranzini in stampa). Ora, è chiaro che anche l'ordine OVS non serve sempre a identificare la fonte di un'informazione proposta in precedenza. Questa funzione è però molto frequente.
} 
presunto ricatto ai suoi danni da parte di Tarantini e Lavitola. Lo dice il legale Piero Longo, interpellato telefonicamente (2011)

(18) (ANSA) - ROMA, 6 SET - "Non so se il governo cadrà sulla empasse [sic] delle pensioni, perché si regge su patti di potere non trasparenti. Se Berlusconi non si rende conto che la cosa migliore che può fare è andarsene, il crollo di credibilità rischia di travolgere il Paese". Lo ha detto il presidente del Copasir, Massimo D'Alema, unendosi al corteo degli operai di Genova che protesta contro la manovra. (2011)

(19) ROMA (ITALPRESS) - Una perturbazione di origine atlantica in arrivo dalla serata di oggi sulla penisola italiana apportera' un notevole peggioramento delle condizioni meteo a partire dalle regioni nord-occidentali ed in estensione al resto del nord e del centro, accompagnata da un significativo rinforzo dei venti, specie sulle aree di ponente. Lo rende noto il Dipartimento della Protezione civile, che ha emesso un avviso di condizioni meteorologiche avverse, che prevede, per la serata di oggi, precipitazioni a prevalente carattere di rovescio o temporale anche di forte intensità, prima sul nord ovest poi sul resto delle regioni settentrionali in estensione verso il centro Italia e la Sardegna. (17.9.2011)

(20) (ANSA) - COPENHAGEN, 7 SET - Sono stati liberati i sette ostaggi danesi che da sei mesi erano tenuti prigionieri da pirati somali. Lo ha reso noto il ministero degli Esteri di Copenhagen. (2011)

(21) (ANSA) - MOSCA, 7 SET - Un aereo di linea russo Yak-42 si è schiantato al decollo a Yaroslav, circa 300 chilometri a nord est di Mosca, causando la morte di 36 persone. Lo scrive l'agenzia Interfax. Tra le vittime, ci sarebbe l'intera squadra di hockey del Lokomotiv Iaroslav, affermano le fonti. È ancora in vita una delle 37 persone, fra passeggeri e personale di equipaggio, che si trovavano a bordo dell'aereo. (2011)

(22) $\left[\#^{16}\right]$ Sale a 32 il bilancio dei civili uccisi oggi in Siria in diverse località dalle forze di sicurezza intervenute per disperdere manifestazioni anti-regime. Lo riferiscono $i$ Comitati di coordinamento locali, una delle piattaforme del movimento di protesta, citate dalla tv panaraba al Jazira. (ATS, 16.9.2011)

${ }^{16}$ Il cancelletto indica talvolta che il testo riportato si colloca all'inizio del lancio, subito dopo il titolo. Da notare ancora che riportiamo sempre il testo del lancio nel modo più fedele possibile, senza cioè correggerne gli eventuali errori (per una tipologia degli errori che si trovano nei lanci e che sono dovuti alla rapidità di codificazione e di trasmissione di questi testi si rinvia a De Cesare / Baranzini, in stampa). 
La fonte dell'informazione riportata nel lancio può anche essere codificata all'interno di altre strutture sintattiche, per esempio all'interno di una clausola con ordine non marcato dei costituenti e con soggetto nullo:

(23) [\#] Le forze del Cnt si ritirano da Bani Walid sotto il pesante lancio di razzi dei lealisti. É quanto ha verificato la Reuters sul posto. (ATS, 16.9.2011)

5.2.2. Nei lanci dell'agenzia TMNews, la cui dimensione è molto variabile (alcuni testi si compongono di un solo capoverso, altri ne contano più di cinque, il che si verifica in parte solo nei lanci dell'agenzia svizzera ATS), le costruzioni scisse svolgono anche altre funzioni: si osservano in particolare, oltre alla funzione che si associa classicamente a questa struttura, ovvero quella contrastiva o correttiva, la funzione di transizione o di costruzione del testo, espressa da frasi scisse il cui elemento segmentato coincide con un'anafora, che riprende in generale un referente saliente oppure che incaspula un'ampia porzione del testo precedente. Ecco un esempio paradigmatico, che contiene due frasi scisse, la prima con funzione correttiva, la seconda con funzione coesiva:

(24) Roma, 15 set. (TMNews) - Sarà anche perchè si tratta del nono processo che, direttamente o indirettamente, lo chiama in causa. O forse perché, come dice il suo avvocato Niccolò Ghedini, "non ci sono dubbi sull'assoluzione". Fatto sta che di fronte alla decisione del gip di Milano di respingere la richiesta di archiviazione per la fuga di notizie sulla telefonata Fassino-Consorte nel caso Unipol-Bnl, il presidente del Consiglio Silvio Berlusconi avrebbe reagito con un atteggiamento a metà tra lo stizzito e la scrollata di spalle. "Ma ti pare avrebbe detto a un suo interlocutore - che io sono oggetto di 100mila intercettazioni e per una volta che io parlo di una intercettazione chiedono l'imputazione?". Per il premier, infatti, la mossa del gip di Milano, che a suo giudizio è arrivato addirittura a 'sconfessare' la posizione dei pm "che notoriamente non sono dalla mia parte", non è altro che la dimostrazione del tentativo di usare la giustizia per sovvertire il voto popolare. Dunque lui.

Non è quindi la 'novità' che arriva dal capoluogo lombardo a tenere sulle spine il presidente del Consiglio, quanto lo spettro di un accerchiamento e soprattutto gli altri due filoni giudiziari: quello napoletano, che secondo i pm lo vede vittima di un'estorsione da parte del tandem Lavitola-Tarantini, e quello barese in cui lo stesso 'Gianpi' è indagato per un giro di prostituzione. Ed è a questa inchiesta che il premier fa riferimento quando parla di 100mila intercettazioni. (2011) 


\section{LE COSTRUZIONI SCISSE NEI LANCI IN ITALIANO E IN INGLESE}

\subsection{Dati quantitativi e forma delle costruzioni scisse}

6.1.1. Partiamo dal caso delle frasi scisse. I dati quantitativi assoluti (cioè il numero di occorrenze rinvenute nel nostro corpus) e relativi (il numero di occorrenze allineato a 100.000 parole) sono riportati nella Tabella 3:

\begin{tabular}{|l|c|c|}
\hline & It & Ing \\
\hline N di occ. & 14 & 8 \\
\hline N di occ. per 100'000 & 23 & 15 \\
\hline
\end{tabular}

Tabella 3. Frequenza delle frasi scisse nei lanci in italiano e in inglese

I dati contenuti in questa tabella permettono di osservare che nel corpus di lanci in italiano ci sono più frasi scisse che in quello in inglese (23 vs 15). La differenza tra i dati dell'italiano e quelli dell'inglese potrebbe essere spiegata - oltre che sulla base del tipo di funzione sintattica svolta dall'elemento scisso, su cui non diremo nulla di preciso qui - a partire dai contesti in cui occorrono le scisse: nei lanci in italiano le scisse compaiono meno di frequente all'interno del discorso riportato. Vi sono infatti 3 frasi scisse su 14 nel discorso riportato (21\%), mentre che se ne trovano 4 casi su 8 (ovvero il 50\%) nei lanci in inglese. Ecco alcuni esempi:

(25) Ho responsabilità politiche, ma non fui io a uccidere (titolo, ANSA, 7.9.2011)

(26) Berlusconi: "Il debito? Sostenibile" "È l'opposizione che ci rovina" (titolo, Adnkronos, 13.9.2011)

(27) Most men down there are pretty macho and they would say things like 'he's scared of his wife and doesn't dare to open his mouth'. They would think that it was the wife who had decided on something like that. (AP, 23.10.2011)

(28) "The kids get along great, it's the adults who are the problem", Warren said. (AP, 22.10.2011)

Rispetto alla frase scissa dell'italiano, la cleft sentence sembra dunque essere legata molto più fortemente all'oralità che non al testo del giornalista. Questo dato può essere confermato alla luce delle osservazioni proposte nella grammatica corpus-based della lingua inglese (senza distinzione diatopica tra inglese britannico e inglese americano) di Biber et al. (1999): nel parlato, ci sono 40 occorrenze di itcleft per 100.000 parole, mentre che se ne trovano solo la metà nella prosa giornalistica (un numero, si noterà, molto simile a quello che abbiamo dato per i 
lanci di agenzia) ${ }^{17}$. Si tratta qui ovviamente solo di una prima ipotesi esplicativa (per una spiegazione più dettagliata e approfondita della diversa frequenza d'impiego delle frasi scisse nei testi giornalistici online in italiano e in inglese, rimandiamo a Garassino, in prep.).

6.1.2. Veniamo alle costruzioni scisse con focus finale, ovvero alle scisse inverse (che si trovano in italiano ma non in inglese) e alle pseudoscisse in senso stretto (che includono le pseudoscisse classiche, aperte in italiano dai pronomi semplici o doppi chi, quello che, ciò che ecc., e in inglese soprattutto dai pronomi what e all), così come alle pseudoscisse inaugurate da un $\mathrm{SN}$ la cui testa coincide con un nome generico: la persona che..., the one who...), alle quali abbiamo affiancato una serie di pseudoscisse dallo statuto più discusso, che sono quelle aperte da un SN la cui testa coincide con un nome semanticamente più ricco (cfr. other areas dell'esempio (35) proposto sotto $)^{18}$. Le costruzioni scisse a focus finale di cui abbiamo tenuto conto in questo lavoro possono essere illustrate a partire dal campione di esempi seguenti:

(29) a fare le spese della situazione attuale è il Ticino (ATS, 16.9.2011, scissa inversa)

(30) Quelli che vanno per la maggiore, ha aggiunto, sono gli spermatozoi di donatori con capelli castani e occhi scuri (ATS, 17.9.2011, pseudoscissa aperta da un pronome)

(31) la cosa migliore che può fare è andarsene (ANSA, 6.9.2011, pseudoscissa aperta da un SN pieno)

(32) il primo a congratularsi con Maroni per il voto su Papa fu Italo Bocchino (TMNews, 15.9.2011, pseudoscissa aperta da un SN pieno)

(33) what I think he was doing was voicing and expressing anger (AP, 22.10.2011, pseudoscissa aperta da un pronome, detta wh-cleft)

(34) All it takes is one pregnant female to survive (AP, 22.10.2011, pseudoscissa aperta da un pronome, detta all-cleft)

\footnotetext{
${ }^{17}$ D'altra parte, come ha notato Katz 2000, e come mostra di nuovo l'ampia ricerca corpus-based di Biber et al. 1999, le it-clefts dell'inglese hanno anche un marcato carattere formale: se ne trovano 60 occorrenze per $100^{\prime} 000$ parole nella prosa accademica.

${ }^{18} \mathrm{Su}$ questa questione, relativamente spinosa, rinviamo alle osservazioni proposte in Collins 1991 e in Calude 2009 sulla lingua inglese, ma che si possono benissimo estendere ai dati dell'italiano.
} 
(35) Other areas where they are pushed into in the red are health bills (SFr254) and online shopping (SFr106). (ATS, 12.9.2011, pseudoscissa aperta da un SN pieno)

Ecco adesso i dati quantitativi relativi alla frequenza d'impiego delle costruzioni scisse a focus finale nel nostro corpus di lanci:

\begin{tabular}{|c|c|c|}
\hline & It & Ing \\
\hline N di occ. & $24^{19}+5=29$ & 12 \\
\hline N di occ. per 100'000 & $40+8=48$ & 23 \\
\hline
\end{tabular}

Tabella 4. Frequenza delle scisse a focus finale nei lanci in italiano e in inglese

I dati contenuti nella Tabella 4 permettono di osservare che nei lanci in italiano vi sono nettamente più costruzioni scisse a focus finale che in quelli in inglese. Da questa tabella risulta anche evidente che l'italiano e l'inglese non usano le stesse costruzioni scisse a focus finale. Nei lanci in italiano compaiono soprattutto le frasi scisse inverse (verbali e nominali, di cui si trovano rispettivamente 19 e 5 occorrenze nel corpus, il che ammonta a 40 occorrenze per 100.000 parole), le frasi pseudoscisse sono invece molto più rare (ne abbiamo trovato solo 5 casi); da notare anche che nel nostro corpus non c'è nessun caso di pseudoscissa aperta da un pronome wh (chi, dove ecc.): tutte le pseudoscisse rinvenute nel corpus hanno una delle forme esemplificate ai punti (30)-(32). Nei lanci in inglese, al contrario, la pseudoscissa aperta da un pronome wh- è una delle forme più ricorrenti (ve ne sono 5 occorrenze su 12); un'altra forma ricorrente di pseudoscissa è quella con SN pieno (di nuovo 5 occorrenze su 12); vi sono poi alcuni casi di pseudoscissa aperta da all (2 occorrenze su 12).

\subsection{Funzioni delle costruzioni scisse}

6.2.1. Come abbiamo già rilevato nel $\S 5$, nei lanci una delle funzioni più caratteristiche delle costruzioni scisse all'interno dei lanci, in particolar modo delle scisse inverse, consiste nel riportare la fonte di un'informazione data in precedenza, caratteristicamente all'inizio del lancio. Eccone di nuovo un esempio paradigmatico:

(36) Parigi, 9 set. (Adnkronos/Dpa) - "Coloro che ci hanno attaccato 1'11 settembre, volevano scavare un fossato tra gli Stati Uniti ed il resto del mondo. Hanno fallito". A scriverlo, commentando la ricorrenza degli attentati di 10

\footnotetext{
${ }^{19}$ Questo primo dato numerico è relativo alle sole scisse inverse.
} 
anni fa, è il presidente americano Barack Obama, in un intervento su 'Le Figaro’. (2011)

Nei lanci in inglese, invece, le costruzioni scisse - in particolare le frasi pseudoscisse, che sono le forme più vicine alle scisse inverse dell'italiano - non adempiono mai alla funzione di riporto della fonte delle informazioni proposte sotto forma di discorso riportato. In modo simile alle pseudoscisse dell'italiano, le pseudoclefts si trovano piuttosto all'interno del discorso riportato (si tratta di $83 \%$ delle occorrenze rinvenute nei lanci in inglese e di $60 \%$ di quelle trovate nei lanci in italiano). La funzione delle pseudoclefts consiste piuttosto nel valutare o modalizzare (anche attraverso la semplice espressione dell'illocuzione effettuata) il proprio dettato, secondo una modalità che è stata descritta da ultimo in Gast \& Wiechmann (2012): la prima parte della pseudoscissa contiene il commento metatestuale, la seconda il contenuto commentato e modalizzato. Ecco alcuni esempi rappresentativi, nei quali si osserva che la pseudoscissa coincide con un meccanismo sintattico che permette di staccare in modo netto la parte di commento (what I think he was doing / what I'm saying) dalla parte commentata:

(37) Really, what I think he was doing was voicing and expressing anger, that he was of no danger to anybody, but was actually supporting people who are trying to make it so the U.S. would stop doing what our military was doing in the Arab world. (AP, 22.10.2011)

(38) "What I'm saying is it ultimately gets down to a choice that that family or that mother has to make", Cain told CNN host Piers Morgan. (AP, 23.10.2011)

Nel nostro corpus di lanci in inglese la fonte di un'informazione riportata all'inizio del lancio (quando viene fornita e quando il lancio si apre effettivamente con una citazione) non viene mai identificata via l'elemento segmentato di una costruzione scissa, ma attraverso altre strategie sintattiche, quali per esempio il soggetto di una clausola con ordine non marcato dei costituenti (39) oppure ancora, come in (40), quello di una clausola marcata con ordine dei costituenti OSV (dove $\mathrm{O}$ coincide con la citazione, $\mathrm{S}$ con la fonte della citazione e $\mathrm{V}$ con un verbo di dire):

(39) NAIROBI, Kenya (AP) - A Kenyan suspect arrested after two grenade blasts rattled Nairobi this week said in court on Wednesday that he took part in one of the attacks and that he is a member of the al-Qaida-linked Somali militant group al-Shabab. (26.10.2011)

(40) BEIRUT (AP) - Senior Arab officials visiting Syria pressed President Bashar Assad on Wednesday to start a dialogue with the opposition, hours after tens of thousands packed a Damascus square to show support for their embattled leader, state TV reported. (26.10.2011) 
La seconda modalità di riporto della fonte di un'informazione incipitaria (ma non solo), illustrata in (40), è molto più vicina a quella illustrata ai punti (17)-(22) per l'italiano, cioè ai casi in cui la fonte viene identificata da un soggetto postverbale di una clausola con ordine dei costituenti OVS. Tra le varie modalità di riporto della fonte di un'informazione precedente - frase scissa inversa e frase non scissa con ordine dei costituenti SVO / OSV / OVS - vi sono delle differenze importanti anche a livello informativo: una fonte identificata attraverso l'elemento segmentato di una frase scissa inversa è molto più saliente di una fonte identificata via il soggetto di una clausola con ordine SVO / OSV (inglese) o OVS (italiano) dei costituenti. La soluzione con la scissa inversa presenta infatti la fonte in modo molto più indipendente rispetto all'informazione citata: la fonte viene riferita nell'ambito di un enunciato autonomo, mentre che nelle clausole con ordine SVO / OSV / OVS fonte e citazione fanno parte di un unico enunciato.

\section{OSSERVAZIONI CONCLUSIVE}

Alla luce delle osservazioni quantitative e qualitative proposte in questo lavoro, in particolare nell'ambito dei paragrafi 5 e 6 dedicati alla descrizione della frequenza, della forma e delle funzioni delle costruzioni scisse in un corpus di lanci di agenzia scritti in italiano e in inglese, si può ora tentare di fornire delle risposte alle quattro domande poste nella parte introduttiva.

(i) Le costruzioni scisse sono già presenti nei testi dei lanci in italiano, cioè nei testi che fondano la lingua dei mass media (giornali cartacei, giornali online, lingua radio-televisiva). Rimane da sapere se queste costruzioni sono impiegate in misura uguale, nelle stesse forme e con le stesse funzioni nei lanci e negli altri testi massmediatici. A questa domanda si potrà però fornire una risposta adeguata solo alla luce di una più ampia ricerca corpus-based, che include testi giornalisti cartacei, online ecc.

(ii) La scrittura dei lanci non è uniforme per quanto riguarda l'impiego delle costruzioni scisse: varia in particolare la loro frequenza d'uso. Questa osservazione può essere collegata in parte, come si suggeriva, alla lunghezza dei testi. Le scisse sono più frequenti nei lanci dell'agenzia TMNews, i cui testi sono appunto più lunghi di quelli delle altre agenzie. Ed è proprio nei lanci di questa agenzia che le scisse svolgono anche importanti funzioni coesive. Questa spiegazione non regge però per i lanci dell'agenzia ATS, che diffonde anch'essa dei testi relativamente lunghi, ma quasi del tutto sprovvisti di costruzioni scisse (lo stesso si può osservare per $\mathrm{i}$ lanci in inglese, in particolare quelli dell'agenzia AP, in generale molto più lunghi dei lanci in italiano, ma che contengono meno scisse).

Di queste costruzioni varia anche il momento della loro manifestazione testuale: le si trova sia nel testo del giornalista che all'interno del discorso riportato in modo 
diretto. In generale, però, queste costruzioni occorrono molto più spesso nel testo del giornalista $(81 \%)$ che nel discorso riportato $(19 \%)$.

Per quanto riguarda la forma delle costruzioni scisse che compaiono nel nostro campione di lanci in italiano va dapprima osservato che vi sono occorrenze non solo di frasi scisse vere e proprie, ma anche di frasi pseudoscisse e soprattutto di frasi scisse inverse. Va poi osservato che quest'ultima costruzione svolge in modo quasi fisso una funzione basica per questo tipo di testo, appartenente - come si era detto alla macrocategoria del testo informativo. Si tratta della funzione di riporto della fonte a monte dell'informazione riferita nel lancio (tipicamente all'inizio, ma anche dopo). Nei lanci in italiano, tuttavia, questa funzione è svolta in modo privilegiato da un'altra struttura sintattica, basata sull'ordine dei costituenti OVS. Rispetto alle clausole OVS, le scisse inverse si presentano dunque come delle strutture sia sintatticamente che funzionalmente più marcate.

(iii) Per quanto riguarda l'impiego delle costruzioni scisse nei lanci in italiano e in inglese, abbiamo osservato che ci sono delle differenze importanti prima di tutto a livello di frequenza d'uso: nei lanci in italiano, sia le scisse classiche sia le scisse a focus finale sono più frequenti che nei lanci in inglese (complessivamente vi sono 72 occorrenze di scisse per 100.000 parole nei lanci in italiano, di contro a 38 in quelli in inglese).

Tra l'italiano e l'inglese vi sono anche delle differenze importanti per quanto riguarda la comparsa delle costruzioni scisse all'interno del discorso riportato (diretto). Rispetto all'inglese, in italiano, sia le scisse che le pseudoscisse sono meno fortemente associate al discorso diretto. Nei lanci in italiano, $21 \%$ delle frasi scisse vere e proprie e $60 \%$ delle frasi pseudoscisse compaiono all'interno del discorso riportato. Nei lanci in inglese, invece, $50 \%$ delle it-clefts e $83 \%$ delle $w h$ clefts si trovano in una citazione.

Un'altra differenza importante tra l'impiego delle costruzioni scisse nei lanci in italiano e in quelli in inglese si verifica a livello funzionale: nei lanci in italiano le scisse (soprattutto quelle di tipo inverso) sono impiegate in modo privilegiato per identificare la fonte di un'informazione riportata sotto forma di citazione all'inizio del lancio.

(iv) Alla luce di quanto detto al punto (iii), ci sembra dunque che l'ipotesi di un possibile influsso dell'inglese sull'italiano - attraverso la presenza di porzioni di testo tradotti dall'inglese in italiano in modo non dichiarato - sia molto dubbia per quanto riguarda l'impiego delle costruzioni scisse nei lanci di agenzia: diversa la frequenza d'impiego di queste costruzioni, sia per quanto riguarda la categoria complessiva, sia i suoi singoli tipi (scisse vere e proprie, pseudoscisse); diverse le forme impiegate, diverse le funzioni e diversi i momenti del testo in cui occorrono queste forme. A partire da queste osservazioni pare licito concludere che l'ipotesi della diffusione delle costruzioni scisse nell'italiano giornalistico odierno a partire da una contaminazione dell'inglese a livello dei testi prodotti in italiano dalle agenzie di stampa sembra poco attendibile. 


\section{RIFERIMENTI BIBLIOGRAFICI}

ALFIERI, Gabriella; CONTARINO, Simona, MotTA, Daria (2003): «Interferenze fraseologiche nel doppiaggio televisivo: l'italiano di E.R. e di Beautiful», in A.V. Sullam Calimani (a c. di), Italiano e inglese a confronto, Firenze, Cesati, pp. 127-149.

ANTONELLI, Giuseppe (2007): L'italiano nella società della comunicazione, Bologna, Il Mulino.

BECCARIA, Gian Luigi (2010): Il mare in un imbuto. Dove va la lingua italiana, Torino, Einaudi.

BENINCÀ, Paola (1978): «Son tre ore che ti aspetto», Rivista di Grammatica Generativa 3, pp. 231-245.

BerRetTA, Monica (1994a): «Ordini marcati dei costituenti di frase in italiano. La frase scissa», Vox Romanica 53, pp. 79-105.

BERRETTA, Monica (1994b): «Il parlato italiano contemporaneo», in L. Serianni e P. Trifone (a c. di), Storia della lingua italiana. III Scritto e parlato, Torino, Einaudi, pp. 239-70.

BERRETTA, Monica (1996): «Come inseriamo elementi nuovi nel discorso/III: Che mi fa paura è la nebbia», Italiano \& Oltre XI, pp. 116-122.

BERRETTA, Monica (2002): "Quello che voglio dire è che: le scisse da strutture topicalizzanti a connettivi testuali», in G. L. Beccaria e C. Marello (a c. di), La parola al testo. Scritti per Bice Mortara Garavelli, Alessandria, Edizioni dell'Orso, pp. 15-31.

BERRUTO, Gaetano (1987): Sociolinguistica dell'italiano contemporaneo, Roma, Carocci.

BIBER, Douglas; JoHANSSON, Stig; LEECH, Geoffrey; CONRAD, Susan; FinEGAN, Edward (1999): Longman Grammar of Spoken and Written English, London, Longman.

BOMBI, Raffaella (2005): La linguistica del contatto - Tipologie di anglicismi nell 'italiano contemporaneo e riflessi metalinguistici, Roma, Il Calamo.

BonOMI, Ilaria (2002): L'italiano giornalistico. Dall'inizio del '900 ai quotidiani on line, Firenze, Cesati.

BRIANTI, Giovanna (2010): «La perifrasi progressiva in progress: confronto tra italiano e inglese», in M. A. Terzoli, A. Asor Rosa e G. Inglese (a c. di), Letteratura e filologia fra Svizzera e Italia. Studi in onore di Guglielmo Gorni. vol. III Dall'Ottocento al Novecento: letteratura e linguistica, Roma, Edizioni di Storia e letteratura, pp. 351-362.

CALARESU, Emilia (2006): «L'universalità del linguaggio scientifico fra norma d'uso e sistema linguistico. Plurilinguismo e monolinguismo nella comunicazione scientifica», in E. Calaresu, C. Guardiano e K. Hölker (a c. di), Italienisch und Deutsch als Wissenschaftssprachen. Bestandsaufnahmen, Analysen, Perspektiven, Münster, Lit Verlag, pp. 29-64. 
CALARESU, Emilia (2011): «The declining status of Italian as a language of scientific communication and the issue of diglossia in scientific communities», International Journal of the Sociology of Language 210, pp. 93-108.

CALUDE, Andrea (2009): Cleft Constructions in Spoken English, Saarbrücken VDM Verlag/Dr. Müller.

Collins, Peter C. (1991): Cleft and Pseudo-cleft Constructions in English, London, Routledge.

CORTElazzo, Manlio (2007): «La perifrasi progressiva in italiano è un anglicismo sintattico?», in Studi in onore di Pier Vincenzo Mengaldo per i suoi settant'anni a cura degli allievi padovani, vol. II, Firenze, SISMEL/Edizioni del Galluzzo, pp. 1753-1764.

D'ACHILLE, Paolo (2003): L'italiano contemporaneo, Bologna, Il Mulino.

D’ACHILle, Paolo; ProietTI, Domenico; VIVIANI, Andrea (2005): «La frase scissa in italiano: aspetti e problemi», in I. Korzen e P. D'Achille (a c. di), Tipologia linguistica e società. Due giornate italo-danesi di studi linguistici (Roma, 27-28 novembre 2003), Firenze, Cesati, pp. 249-79.

DARDANO, Maurizio (1994): «Profilo dell'italiano contemporaneo», in L. Serianni e P. Trifone (a c. di), Storia della lingua italiana, vol. II., Torino, Einaudi.

DE CESARE, Anna-Maria (2005): «La frase pseudoscissa in italiano contemporaneo. Aspetti semantici, pragmatici e testuali», Studi di grammatica italiana XXIV, pp. 293-322.

DE CESARE, Anna-Maria (2009): «La posizione del soggetto tra scritto e parlato: un'analisi alla luce dei casi 'anche + soggetto", in A. Ferrari (a c. di), Atti del X Congresso SILFI. Sintassi storica e sincronica, Subordinazione, coordinazione, giustapposizione. Basilea, 30 giugno-3 luglio 2008, Firenze, Franco Cesati Editore, pp. 955-970.

DE CESARE, Anna-Maria (2010): «La scrittura italiana odierna, tra modi dell'italiano parlato e della scrittura anglo-americana», in A. Ferrari e A.-M. De Cesare (a c. di), Il parlato nella scrittura italiana odierna. Riflessioni in prospettiva testuale, Frankfurt a/M, Lang, pp. 33-55.

DE CESARE, Anna-Maria (2011): L'ordine dei costituenti in italiano contemporaneo e in prospettiva contrastiva con il tedesco. Tra sintassi, pragmatica e tipologia linguistica, Habilitationsschrift, Istituto d'italianistica, Università di Basilea, MS.

De Cesare, Anna-Maria (in prep.): «Cleft Constructions in Italian and in a contrastive perspective: definition, taxonomy, examples, problems», in A.-M. De Cesare (a c. di), Frequency, forms and functions of Cleft Constructions in Romance and Germanic. Contrastive, corpus-based studies, Berlin, De Gruyter/Mouton.

DE CESARE, Anna-Maria; BARANZINI, Laura (in stampa): «La variété syntaxique des dépêches d'agence publiées en ligne. Réflexions à partir d'un corpus de langue italienne», in A. Ferrari e L. Lala (a c. di), Variétés syntaxiques dans la 
variété des textes. Quelques exemples à partir de l'italien contemporain online (=Verbum, II/2011), pp. 247-302.

DEgANO, Chiara (2005): «Influssi inglesi sulla sintassi italiana: uno studio preliminare sul caso della perifrasi progressiva», in A. Cardinaletti e G. Garzone (a c. di), L'italiano delle traduzioni, Milano, FrancoAngeli, pp. 85-105.

FILPPULA, Markku (2009): «The rise of it-clefting in English: areal-typological and contact-linguistic considerations», English Language and Linguistics 13/2, pp. 267-293.

FORNACIARI, Raffaello (1881): Sintassi italiana dell'uso moderno, Firenze, Sansoni [Ristampa anastatica 1974 a c. di G. Nencioni].

GARASSINO, Davide (in prep.): «Cleft Sentences. Italian-English in Contrast», in A.-M. De Cesare (a c. di), Frequency, forms and functions of Cleft Constructions in Romance and Germanic. Contrastive, corpus-based studies.

GAST, Volker; Daniel WIEChMANN (2012): «W(h)-Clefts im Deutschen und Englischen. Eine quantitative Untersuchung auf Grundlage des EuroparlKorpus», in L. Gunkel e G. Zifonun (a c. di), Jahrbuch des IDS 2011, Berlin, de Gruyter/Mouton.

GIL, Alberto (2004): «Textstrukturelle Dimensionen der Satzspaltung im Italienischen», in A. Gil, D. Osthus e C. Polzin-Hausmann (a c. di), Romanische Sprachwissenschaft. Zeugnisse für Vielfalt und Profil eines Faches. Festschrift für Christian Schmitt zum 60. Geburtstag, vol. II, Frankfurt a/M., Lang, pp. 36985.

GRASSO, Daniele (2007): Innovazioni sintattiche in italiano alla luce della nozione di calco, Tesi di Dottorato, Ginevra.

GuALDO, Riccardo (2007): L'italiano dei giornali, Roma, Carocci.

JESPERSEN, Otto (1937): Analytic Syntax, London, Allen and Unwin.

KATZ, Stacey (2000): "Categories of c'est-cleft constructions», Canadian Journal of Linguistics / Revue canadienne de linguistique 45(3-4), pp. 253-273.

KLAJN, Ivan (1972): Influssi inglesi nella lingua italiana, Firenze, Olschki.

LAMBRECHT, Knud (2001): «A Framework for the Analysis of Cleft Constructions», Linguistics 39(3), pp. 463-516.

LEPRI, Sergio (1982): Le macchine dell'informazione. Ieri, oggi, domani delle agenzie di stampa, Sonzogno, Etas Libri.

MigLIORINI, Bruno ( $\left.{ }^{2} 1963\right)$ : Storia della lingua italiana, Firenze, Sansoni.

MiLlER, Jim (2006): «Focus in the languages of Europe», in G. Bernini e M. L. Schwartz (a c. di), Pragmatic Organization of Discourse in the Languages of Europe, Berlin/New York, de Gruyter, pp. 121-214.

MONDELlO ANZÀ, S. (2002): I fenomeni di sintassi marcata nei quotidiani contemporanei, Tesi di laurea, Università degli Studi di Milano.

PALERMO, Massimo (1995): «I manuali redazionali e la norma dell'italiano scritto contemporaneo», Studi linguistici italiani XXI, pp. 88-115. 
PALERMO, Massimo (1997): «La lingua in agenzia: aspetti della norma e dell'uso dell'ANSA», Norma e lingua in Italia: alcune riflessioni fra passato e presente, Istituto Lombardo di Scienze e Lettere, Milano, pp. 185-205.

PANUNZI, Alessandro (2009): «Strutture scisse e pseudoscisse: valori d'uso del verbo essere e articolazione dell'informazione nell'italiano parlato», in A. Ferrari (a c. di), Atti del X Congresso SILFI. Sintassi storica e sincronica dell'italiano. Subordinazione, coordinazione, giustapposizione (Basilea, 30 giugno-3 luglio 2008), Firenze, Cesati, vol. II, pp. 1121-1137.

PAPUZZI, Alberto (2003): [Nuova ed. completamente riveduta e aggiornata], Professione giornalista. Tecniche e regole di un mestiere, con la collab. d'A. Magone, Roma, Donzelli.

RogGiA, Carlo Enrico (2008): «Frasi scisse in italiano e francese orale: evidenze dal C-ORAL-ROM», Cuadernos de filología italiana 15, pp. 9-29.

RogGiA, Carlo Enrico (2009): Le frasi scisse in italiano. Struttura informativa e funzioni discorsive, Genève, Slatkine.

RogGIA, Carlo Enrico (2012): «Frasi scisse in italiano antico: alcune proposte», in B. Wehr e F. Nicolosi (a c. di), Pragmatique historique et syntaxe / Historische Pragmatik und Syntax. Actes de la section du même nom du XXXIe Romanistentag allemand / Akten der gleichnamigen Sektion des XXXI. Deutschen Romanistentags (Bonn, 27. 9.-1.10. 2009), Frankfurt a/M., Lang, pp. 193-221.

SABATINI, Francesco (1985): “"L'italiano dell'uso medio": una realtà tra le varietà linguistiche italiane», in G. Holtus e E. Radtke (a c. di), Gesprochenes Italienisch in Geschichte und Gegenwart, Tübingen, Narr, pp. 154-184.

SABATINI, Francesco (2011 [1999]), «"Rigidità-esplicitezza" vs "elasticitàimplicitezza": possibili parametri massimi per una tipología dei testi», in V. Coletti et al. (a c. di), L'italiano nel mondo moderno. Saggi scelti dal 1968 al 2009, vol. II, pp. 183-216.

SERIANNI, Luca (1988): Grammatica italiana. Italiano comune e lingua letteraria, Torino, UTET.

SORNICOLA, Rosanna (1994): «On Word-order variability: A study from a Corpus of Italian», Lingua e Stile XXIX/1, pp. 25-57.

SUllam CALIMANI, Anna-Vera (a c. di) (2003): Italiano e inglese a confronto, Firenze, Cesati. 


\section{APPENDICE}

\section{Obama annuncia, la guerra e' finita}

I nostri soldati a casa per feste fine anno

21 ottobre, 19:25

\$ Indietro G G Stampa $\square$ Invia \& Scrivi alla redazione $\square$ Suggeriscio

WASHINGTON - "Oggi posso annunciare quello che era stato promesso": Io ha detto il presidente degli Usa, Barack Obama, annunciando il ritiro totale delle truppe dall'Iraq entro la fine dell'anno.

"Dopo nove anni la guerra in Iraq finira', la marea della guerra si ritira" ha detto il presidente, assicurando come "tutti i nostri soldati torneranno a casa per le feste di fine anno"

(-) Copyright ANSA - Tutti i diritti riservati

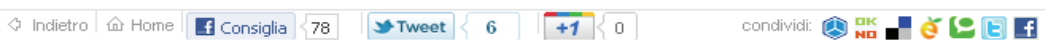

\section{Obama: la guerra in Iraq è finita, le truppe a casa entro l'anno}

Restano circa $\mathbf{4 0 . 0 0 0}$ soldati nel Paese, il presidente: a casa per le feste. In nove anni9 sono 4.400 i militari statunitensi morti

Pubblicato il 21/10/11 da TMNews in Esteri | TAGS: new york
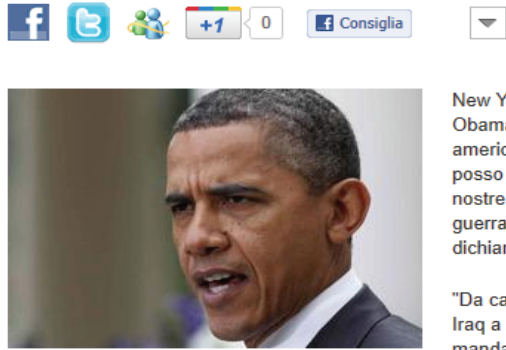

New York, 21 ott. (TMNews) - II presidente Barack

Obama ha annunciato che le restanti truppe americane in Iraq rientreranno entro il 2011. "Oggi posso dire che come ho promesso il resto delle nostre truppe tornerà a casa. Dopo nove anni la guerra in Iraq finirà", ha detto leggendo una dichiarazione alla Casa Bianca.

"Da candidato ho promesso di portare la guerra in Iraq a una fine responsabile. Assumendo il mandato ho annunciato una strategia responsabile per ritirare le truppe entro il 2011. L'anno scorso ho annunciato la fine della nostra missione di combattimento in Iraq", ha detto Obama, annunciando che "poche ore fa ho parlato con il primo ministro Nouri al-Maliki, e siamo in pieno accordo su come procedere".

II presidente non ha accennato alla controversia sulla possibile permanenza di circa 5.000 soldat americani in Iraq come consiglieri, sul cui status legale non c'era però accordo, e che non rimarranno

Le truppe americane "saranno a casa per le feste", ha promesso Obama. I soldati torneranno "fieri del loro successo e sapendo che il popolo americano è unito nel sostenerli".

"In accordo con l'intesa strategica conclusa con l'Iraq, il nostro rapporto sarà una partnership paritetica tra nazioni sovrane", ha detto il presidente.

Attualmente rimangono in Iraq circa 40.000 soldati americani. Nel marzo del 2003, erano stati circa 150.000 i soldati a invadere l'Iraq per deporre il regime di Saddam Hussein; 4.400 sono rimasti uccisi nei quasi nove anni da allora. 


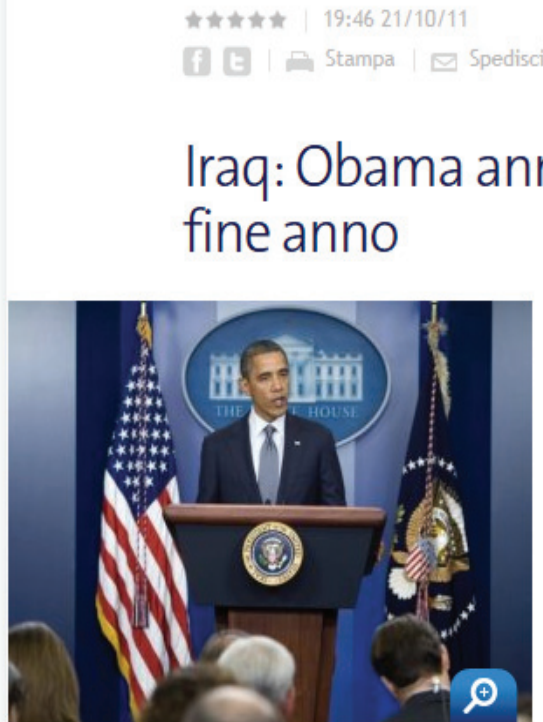

Obama annuncia il prossimo ritiro delle truppe Foto: Keystone

\author{
Il presidente americano Barack Obama \\ ha annunciato il ritiro totale delle \\ truppe Usa dall'Iraq entro la fine \\ dell'anno.

\begin{abstract}
"Oggi posso annunciare quello che era stato promesso", ha detto Obama. "Dopo nove anni la guerra in Iraq finirà, la marea della guerra si ritira", ha aggiunto, assicurando che "tutti i nostri soldati torneranno a casa per le feste di fine anno".

L'annuncio segue una videoconferenza del presidente con il premier iracheno Nuri al Maliki.
\end{abstract}

Nelle ultime settimane si era detto che un numero compreso tra 3.000 e 10.000 soldati americani potrebbero rimanere in Iraq con compiti di addestramento delle forze di sicurezza irachene.

(ats)

Tags Politica

Condividi su... f Facebook $\mid \mathbf{E}$ Twitter $\mid+$ Altri Social Network

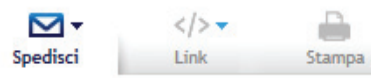


NewS > Esteri > L'annuncio di Obama: "Tutte nostre truppe in Iraq saranno a casa per Natale" "Dopo quasi nove anni la guerra sarà finita"

\section{L'annuncio di Obama: "Tutte nostre truppe in Iraq saranno a casa per Natale"}

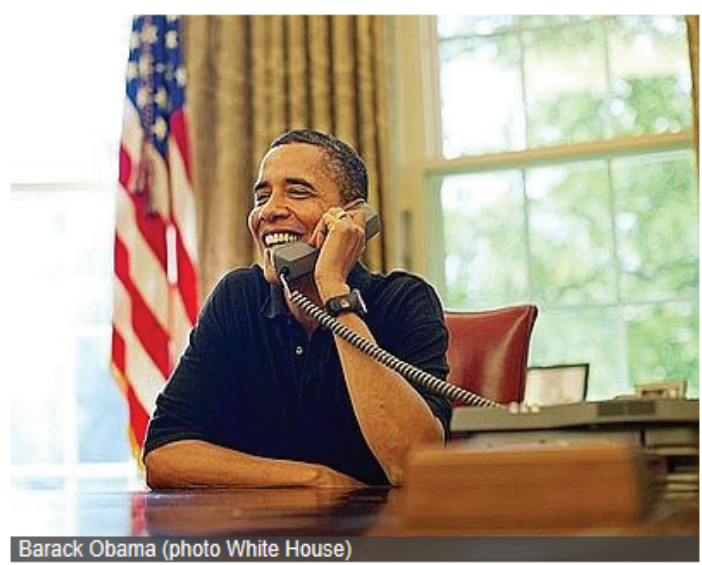

ultimo aggiornamento: 21 ottobre, ore 19:18 Washington - (Adnkronos) - La dichiarazione alla stampa dopo una conversazione telefonica tra il presidente americano e al-Maliki: il ritiro sarà completato entro la fine del 2011. L'inquilino della Casa Bianca: "Mantenuti gli impegni presi"

condividi

commenta 回 0 vota 3 invia stampa

f的 Mi piace \%ั:

Washington, 21 ott. (Adnkronos) - "Tutte le nostre truppe in Iraq saranno a casa per Natale, e dopo quasi nove anni la guerra sarà finita". E' quanto ha detto Barack Obama annunciando che entro la fine del 2011 sarà completato il ritiro delle forze americane in Iraq. II presidente ha rilasciato una breve dichiarazione alla stampa dopo aver avuto un colloquio telefonico con il premier Nouri al-Maliki in cui "ho ribadito che gli Usa mantengono gli impegni presi e come promesso il rimanente delle truppe rimaste rientreranno negli Stati Uniti entro la fine dell'anno",

Anche se l'impegno militare americano in Iraq sta per finire non verrà interrotta "l'enorme relazione tra i due paesi, basata sul reciproco rispetto e comprensione tra due nazioni sovrane". E, ha detto ancora Obama, "continueranno le discussioni" per trovare un accordo che permetta agli Stati Uniti di continuare ad aiutare I'Iraq, soprattutto per quanto riguarda l'addestramento militare, che avrà di fronte a se "ancora giorni difficili".

pubblica questa notizia su: f 